\title{
Radiation and other pathological changes in breast tissue after conservation treatment for carcinoma
}

\author{
A C Girling, A M Hanby, R R Millis
}

\begin{abstract}
Histological changes in breast tissue after radiotherapy were evaluated. Changes in tissue from 17 patients previously treated for breast cancer by surgery and radiotherapy were compared with those seen in a control group treated with surgery alone. Vascular and epithelial changes were seen only in tissue from patients who had received radiotherapy and, therefore, seemed to be relatively specific; stromal changes were seen in both groups and seemed, therefore, to be non-specific. Epithelial atypia is of particular importance as it may be severe and be confused with recurrent malignancy. The presence of other changes associated with radiotherapy, particularly those in vessels, should help to avoid such misdiagnosis.
\end{abstract}

That histological changes occur after radiotherapy is well known, and these have been described in a variety of tissues ${ }^{12}$ including the breast. $^{3-10}$ The changes most often reported are those seen in epithelium, fibroblasts, and blood vessels. Conservation treatment of primary breast cancer by a combination of surgery and radiotherapy is becoming standard practice in many centres. It is therefore important for pathologists to be aware of the changes in mammary tissue which can be produced by radiotherapy as these are likely to be found increasingly often. Changes in the mammary epithelium can result in pronounced atypia and may pose a particular diagnostic problem.

We report here the histological changes seen in a series of patients who, having been treated conservatively for breast cancer, subsequently had further surgery for a recurrent mass in the breast. These changes were compared with those seen in non-irradiated breast tissue from patients with breast cancer treated by surgery alone.

Research Fund

Department of

Clinical Oncology

A C Girling

R R Millis

Department of

Clinical Microscopy,

Guy's Hospital,

London SE1 9RT

A M Hanby

Requests for reprints to:

Dr R R Millis, Imperia

Cancer Research Fund

Department of Clinical

Oncology, Guy's Hospital,

London SE1 9RT

Accepted for publication

20 September 1989

\section{Methods}

In Guy's Hospital breast unit selected patients have been treated conservatively for primary breast cancer since 1981 . This treatment takes the form of local excision with total axillary clearance and a boost radiation dose with iridium implant to the tumour site of $26 \mathrm{~Gy}$ over two days, followed by external beam megavoltage radiotherapy to the breast of 46 Gy over four and a half weeks. Seventeen patients managed in this way developed a recurrent mass in the treated breast and the material from these patients forms the basis of this paper. The clinical and mammographic features of these patients have been reported previously. ${ }^{11}$ In seven of the 17 cases the new lesion represented recurrent malignancy and in 10 it was benign (fat necrosis $n=7$, foreign body granulomatous reaction $\mathrm{n}=2$, and scar tissue $\mathrm{n}=1$ ). Patients with recurrent malignancy were treated by salvage mastectomy and those with benign lesions by excision biopsy. In the former group, therefore, tissue from all four quadrants of the breast was available for histological examination. All the slides were reviewed independently by two pathologists (ACG and AMH).

In 14 of the 17 cases the recurrent lesion was at the same site as the first. In the three cases in which the recurrent mass was in a different quadrant of the breast the lesion proved to be malignant, necessitating salvage mastectomy. Thus in all cases tissue from the site of the iridium implant-that is, adjacent to the original biopsy site-was reviewed. In all cases the original biopsy specimen was assessed as well as the recurrent lesion and, where applicable, the mastectomy specimen. In addition to tissue from the 17 patients described above, slides of the mastectomy specimens from four other patients were reviewed. These patients, all with primary mammary carcinoma, were treated by surgery alone and did not receive radiation treatment. These specimens, together with the pre-treatment specimens from the study patients, served as controls. Tissue immediately adjacent to and including tumour was not evaluated. The pathologists were unaware of the history of the patients when reviewing the slides.

Particular attention was paid to changes which had previously been reported to be associated with radiation treatment. ${ }^{1-5710}$

Table 1 Recurrent lumps which proved to be benign $(n=10)$ : changes seen at site of iridium implant

\begin{tabular}{lc}
\hline Histological feature & No of cases affected \\
\hline $\begin{array}{l}\text { Epithelial changes } \\
\text { Atrophy } \\
\text { Atypia }\end{array}$ & 8 \\
Stromal changes & 9 \\
"Atypical" fibroblasts & 7 \\
Ground substance & \\
Fibrosis & 10 \\
Elastosis & 10 \\
Fat necrosis with cyst formation & 7 \\
Vascular changes & 7 \\
\hline
\end{tabular}


Table 2 Recurrent lumps which proved to be malignant $(n=7)$ : changes at iridium implant site and elsewhere

\begin{tabular}{lll}
\hline & No of cases affected & \\
\cline { 2 - 3 } Histological feature & Implant site & Elsewhere in breast \\
\hline $\begin{array}{l}\text { Epithelial changes } \\
\text { Atrophy } \\
\text { Atypia }\end{array}$ & 6 & 6 \\
$\begin{array}{ll}\text { Stromal changes } \\
\text { "Atypical” fibroblasts }\end{array}$ & 4 & 5 \\
Ground substance & 4 & 6 \\
Fibrosis & 3 & 7 \\
Elastosis & 7 & 7 \\
Fat necrosis with cyst formation & 4 & 0 \\
Vascular changes & 1 & 3 \\
\hline
\end{tabular}

\section{Results}

Changes considered to be consistent with radiation treatment were noted in epithelium, stroma, and blood vessels. A characteristic pattern of fat necrosis was also noted at the site of the iridium implant in some cases. The incidence of these changes, both at the site of the iridium implant and in the remaining breast, is shown in tables 1 and 2 .

Tissue immediately adjacent to or within the tumour-tumour stroma-was not assessed because of the possible confounding effects resulting from local tumour influences.

\section{EPITHELIAL CHANGES}

Two types of change were observed-atrophy and atypia. These were often seen in combination. The former manifested itself as a reduction in size of the normal lobules due both to loss of acini and a reduction in the number of cells in each acinus. This lobular atrophy was associated with intralobular fibrosis. These changes are similar to those seen in normal breast tissue from elderly subjects. Atypia was seen in the luminal epithelial cells occurring occasionally in small ducts (fig 1), but more frequently in lobular acini (fig 2). The cells tended to be larger than normal with, in some cases, an increased nuclear to cytoplasmic ratio, and they had large pleomorphic nuclei and often prominent but small nucleoli. While the nuclei were typically hyperchromatic the chromatin was nevertheless uniform and clumping was absent. In two cases bizarre multinucleate cells were present. The cytoplasm was usually eosinophilic and sometimes vacuolated. The atypical epithelial cells in the acini were particularly striking because of the overall atrophy of the lobules, so that the cells stood out against a bland background. Furthermore, not all the cells in an acinus were affected. The abnormal cells were irregularly disposed, thus showing striking contrast with their smaller neighbours. No mitoses were seen and in no case was there any evidence of epithelial hyperplasia. All the atypical changes were in the luminal cells; none was seen in the basal (myoepithelial) cells.

\section{STROMAL CHANGES}

Changes were seen in both fibroblasts and ground substance.

\section{FIBROBLASTS}

A variety of changes was seen. The nuclei were sometimes hyperchromatic, but sometimes seemed to be paler than normal-that is, hypochromatic. In the latter case the nuclei often had poorly delineated outlines and seemed to be "smudged". These "smudged" nuclei were frequently set in a background of densely collagenised stroma. Nuclear size was variable and some large and even multinucleate forms occurred. Nucleoli were often present.

\section{GROUND SUBSTANCE}

Changes consisted of fat necrosis, fibrosis, and elastosis. A distinctive pattern of fat necrosis
Figure 1 Epithelial atypia within a duct from irradiated breast tissue. Note large stromal fibroblasts.

(Haematoxylin and eosin.)

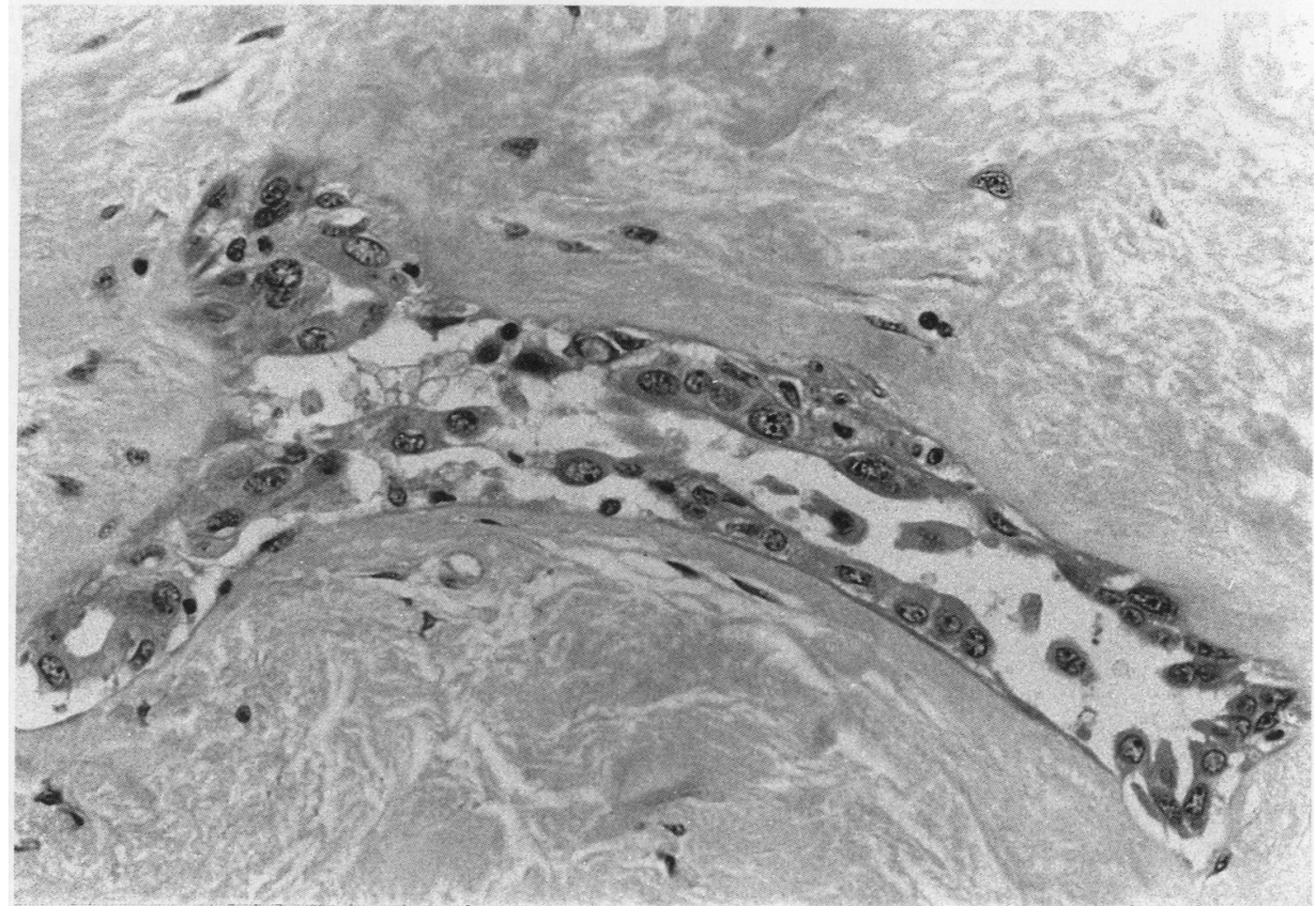




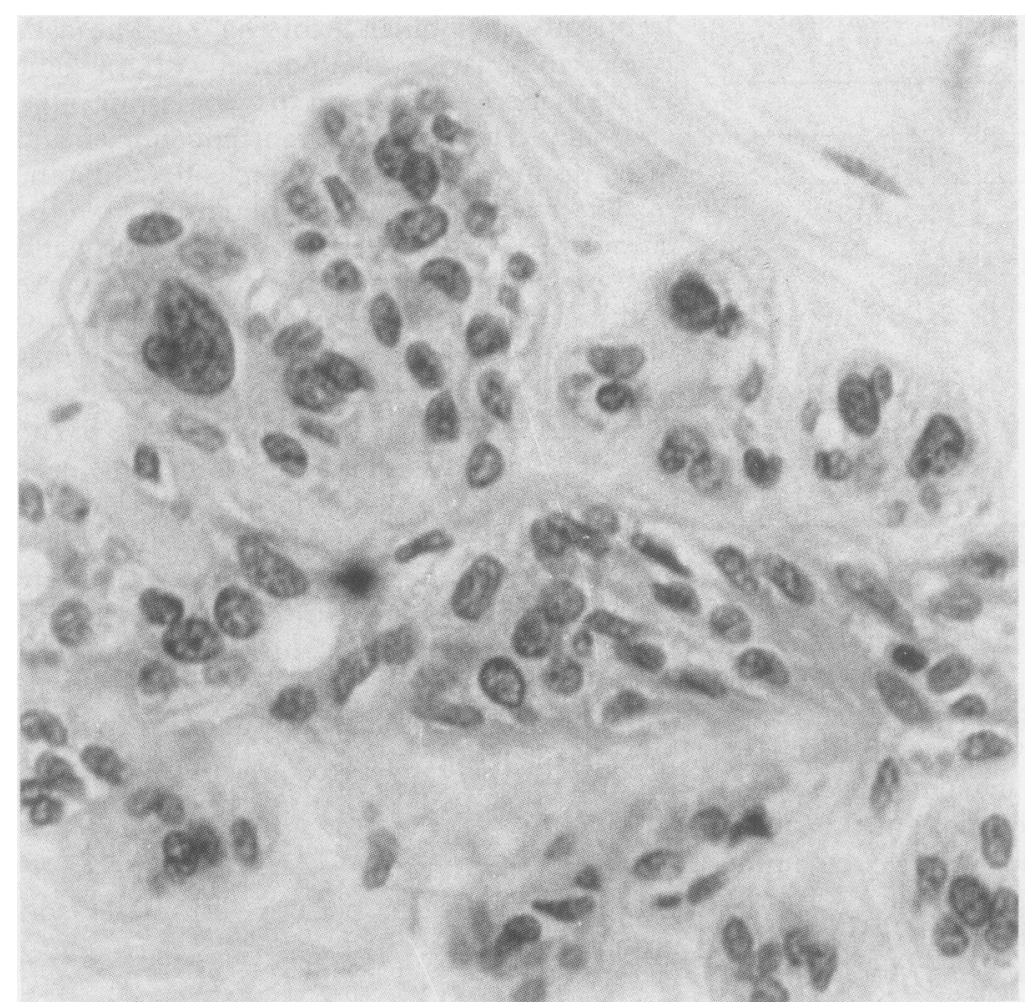

Figure 2 Epithelial atypia within a lobule from irradiated breast tissue. (Haematoxylin and eosin.)

was seen at the site of the iridium implant in many cases, both benign and malignant (fig 3 ). This consisted of an irregular rim of relatively acellular fibrous tissue lined on its inner surface by a layer of fibrin around an area of necrotic fat, giving the overall appearance of a cyst. In one case homogenous eosinophilic material was also seen in the centre of the "cyst". In several cases calcification was present in the fibrous tissue wall. This pattern of fat necrosis was distinct from that commonly seen at biopsy

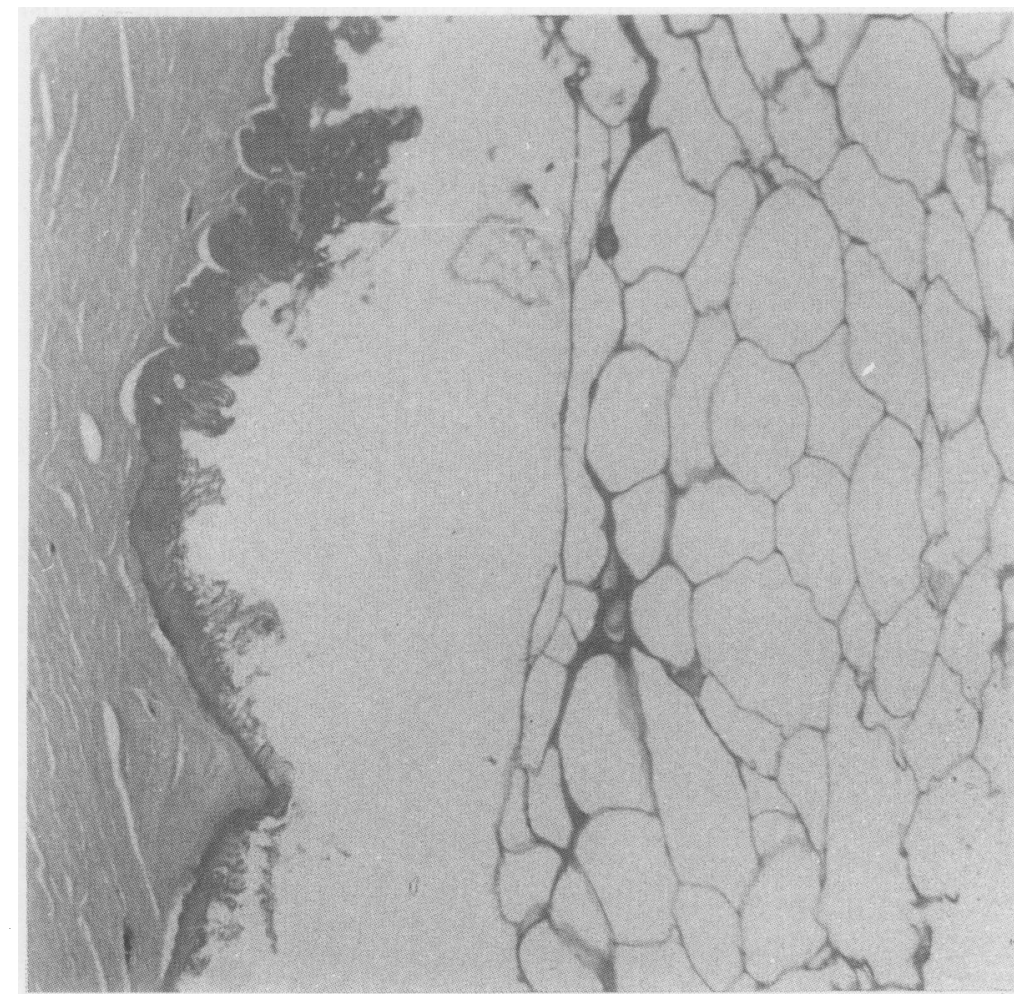

Figure 3 Fat necrosis at site of iridium implant. (Haematoxylin and eosin). sites in mastectomy specimens. Fibrosis occurred as diffuse bands, particularly at the region of the iridium boost (previous biopsy site). An increase in elastic tissue was noted both within the irradiated and non-irradiated fields in vessels and interlobular stroma. Relatively more elastosis was seen in the irradiated breast tissue, though this could not reliably be quantified.

\section{VASCULAR CHANGES}

Changes were observed in arteries and veins of various sizes. In small to medium sized vessels the most common change was fibroblastic intimal thickening (fig 4), sometimes with complete occlusion of the lumen. The elastic lamina was often fragmented with resultant poor definition of the layers of the vessel wall. In small capillaries the endothelial cells often seemed to be particularly prominent. They were frequently larger than normal with hyperchromatic nuclei, which were sometimes vacuolated.

In the original surgical specimens from the study group (before irradiation) and the control mastectomy specimens occasional abnormal fibroblasts were seen (fig 5) similar to those described above, although less striking. Focal areas of elastosis and fibrosis were also sometimes seen in the control mastectomy specimens away from the site of the original biopsy site. No change was noted in the vessels. Particular attention was paid to examination of epithelium in quadrants away from the primary tumour site. Cystic change was observed in three cases and duct ectasia in one, but no epithelial hyperplasia or atypia were seen.

\section{Discussion}

Most of the changes noted in the irradiated tissue, both in the stroma and epithelium of the breast, were similar to those previously described after radiation. ${ }^{-7}$ Connolly and Schnitt, however, who noted similar epithelial changes, found, in contrast to our experience, that atypical fibroblasts and vascular changes were much less common features. ${ }^{10}$

The vascular changes noted, as a whole, seemed to be entirely attributable to radiation, with the changes in the endothelial cells appearing to be relatively specific. Fibroblastic intimal thickening, although occurring in irradiated tissue, can also result from a variety of other tissue insults. Opinion is divided as to the specificity of these vascular changes. ${ }^{4710}$

Fibrosis $^{1-7}$ and elastosis ${ }^{3-5}$ have been extensively described in relation to radiation but, as previously pointed out, ${ }^{10}$ are non-specific changes which can occur as a result of trauma and are also frequently seen in benign breast tissue. In our study it is difficult to know how much of the fibrosis at the iridium boost site can be attributed to radiation as such changes might well result from either surgery or as a reaction- to the implantation of a foreign body. Similarly, the characteristic pattern of fat necrosis in some of the studied cases may be largely due to the physical presence of the implant and have little to do with the radiotherapy. Surprisingly, changes in the 
Figure 4 Vessel from irradiated breast tissue showing intimal thickening and reduplication of elastic laminae. (Orcein).

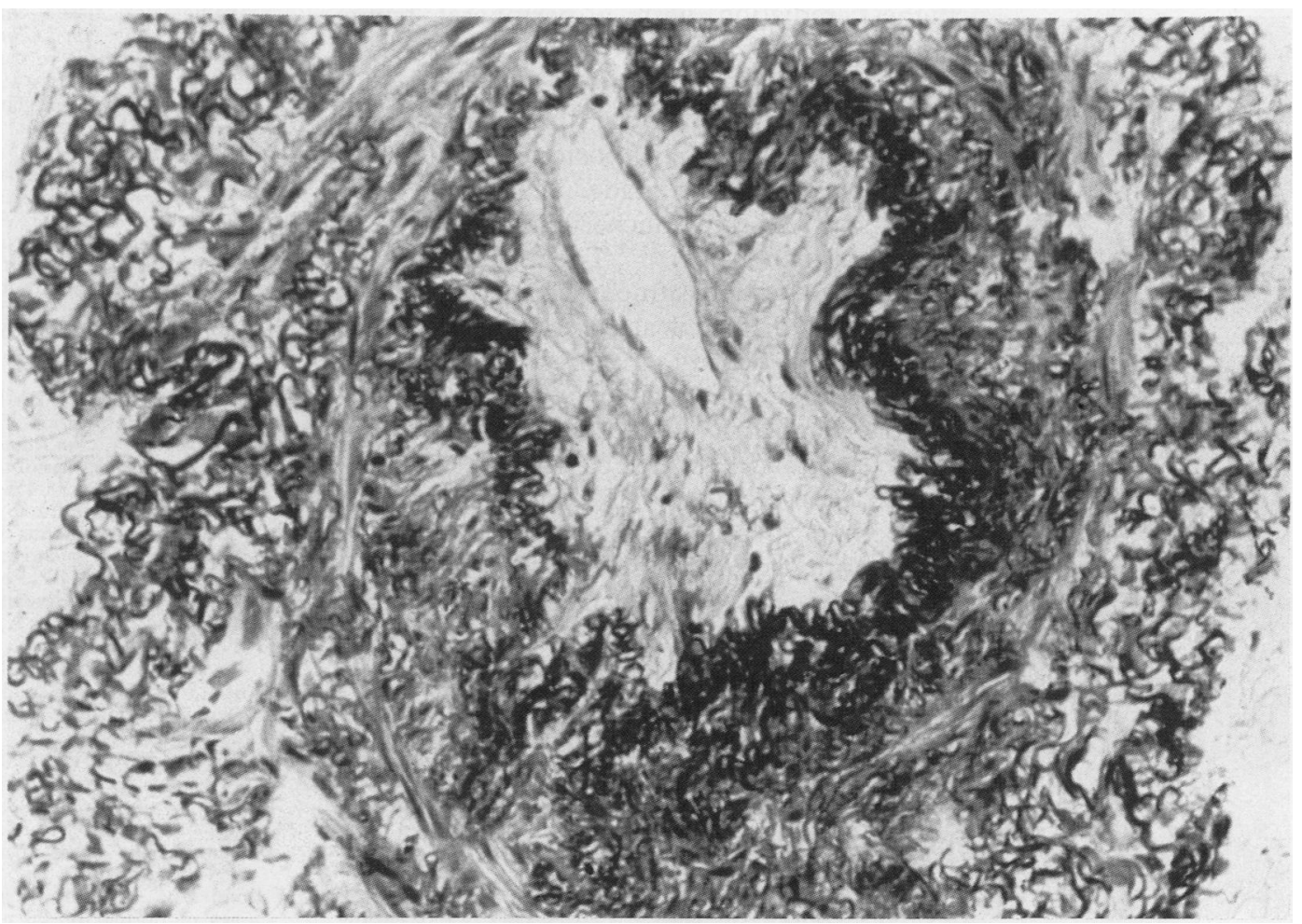

fibroblasts also seemed to be non-specific. Distinct histological appearances that could be attributed solely to radiation were not seen. Similar "atypical" fibroblasts occur in the normal breast ${ }^{12}$ in benign breast disease $\mathrm{e}^{13}$ and also in other tissues, for example, nasal polyps. ${ }^{14}$ We concluded that such cells lie within the normal range of appearance and that the fibroblast is a naturally "pleomorphic cell."

The changes in the epithelium, however, seem to be more specific and, furthermore, may be a source of diagnostic error. These were most severe and worrying at the site of the iridium boost. This problem has been discussed by others, both in histological and cytological material. ${ }^{701516}$ In cytological practice differentiation between atypia resulting from radiation and malignancy is even more difficult because the cells are not seen in the context of the overall mammary architecture. Indeed, on the basis of fine needle aspiration cytology it has been stated that radiation may cause changes in normal epithelium that are

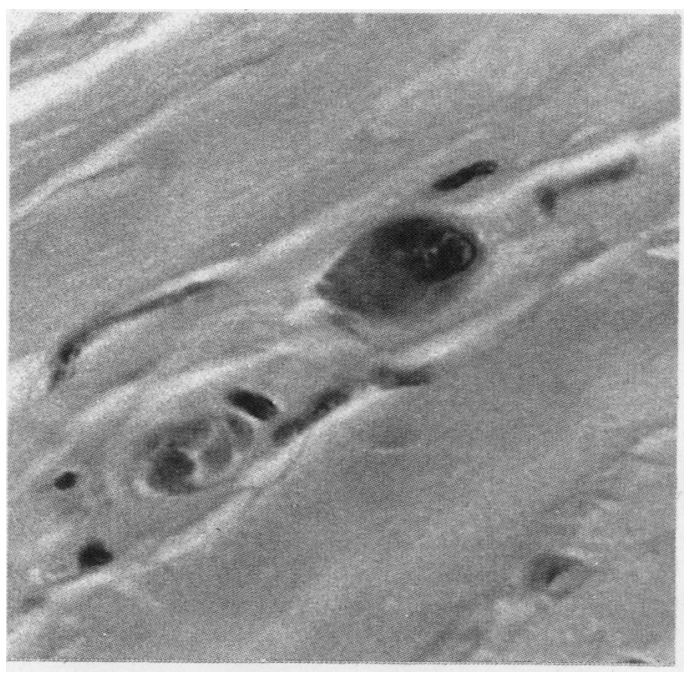

indistinguishable from malignancy. ${ }^{15}$ It has also been claimed, however, that there are certain cytological features which characterise aspirates from non-neoplastic irradiated breast tissue. ${ }^{16}$ These include a normal nuclear to cytoplasmic ratio, despite anisocytosis and anisonucleosis; well preserved nuclei with smooth borders; a regular chromatin pattern and especially the presence of naked bipolar (myoepithelial) nuclei in and around the clusters of cells. On the basis of examination of the histological material in our study we would agree with most of these observations, but occasionally we noted a raised nuclear to cytoplasmic ratio in the benign irradiated cells and feel, therefore, that this feature is not a reliable indicator of malignancy.

In histological material the overall architecture of the lesion was found to be helpful. The most extreme atypia was seen in the acini where the appearance was enhanced by the accompanying lobular atrophy, the very presence of which was useful in distinguishing these changes from malignancy. An additional useful feature was the presence of atypical cells adjacent to unremarkable cells in affected glandular structures. The picture contrasts with the uniform proliferation of atypical cells seen in carcinoma in situ. Other features of in situ carcinoma which, in our experience, are not seen in areas of atypia resulting from radiation, include multilayering of cells with expansion and distension of glandular structures. Similar features have also been found helpful by others. ${ }^{70}$ Differential diagnosis was most difficult when "rafts" of desquamated epithelial cells were present in lumina of small ducts.

It is interesting to observe that some of the changes were found more frequently in the surrounding breast tissue than at the boost site (table 2), though they were often not so severe. This may be due to tissue necrosis at the site of 
highest radiation with a peripheral fringe effect.

Thus in breast tissue treated by conservation, including radiation, there are several stages at which a false positive diagnosis of malignancy can be made. Clinically, local fibrosis with skin puckering may suggest recurrent malignancy. ${ }^{11} 17$ The mammographic appearances after treatment which include skin thickening, distorted architecture, noticeable parenchymal density, the presence of a mass (pseudotumour) and calcification may also be suggestive of this. ${ }^{17}$ Fine needle aspirates may show atypical cells which can also be misinterpreted. And the histological appearance of the epithelium can also simulate malignancy.

It is important that pathologists are aware of the atypical epithelial changes which can occur in irradiated breast tissue and the features which help distinguish them from malignancy. Recognition of the other tissue changes described above, particularly those in blood vessels, should alert clinicians to the possibility of radiation and so avoid a misdiagnosis of malignancy.

The authors extend their thanks to Professor David Levison and Professor Noel Gowing for their helpful advice.

1 Dunlap CE. Radiation injury. In: Anderson JW. Ed. Patho logy. New York: CV Mosby, 1977:253-62.

2 Sindelar WF, Hoekstra H, Restrepo C, Kinsella TJ.
Pathological tissue changes following intra-operative radiotherapy. Am J Clin Oncol 1986;9:504-9.

3 Lumb G. Changes in carcinoma of the breast following irradiation. BrJ Surg 1950;38:82-93.

4 Williams IG, Cunningham GJ. Histological changes in irradiated carcinoma of the breast. $\mathrm{Br} J$ Radiol 1951;24:123-33.

5 Gowing NFC. Histological changes in response to therapy. In: Hayward JL, Bulbrook RD, eds. Clinical evaluation in breast cancer. London: Academic Press, 1966:53-61.

6 Clarke D, Curtis JL, Martinez A, Fajardo L, Goffinet D. Fat necrosis of the breast simulating recurrent carcinoma after primary radiotherapy in the management of early stage primary radiotherapy in the management

7 Schnitt SJ, Connolly JL, Harris JR, Cohen RB. Radiationinduced changes in the breast. Hum Pathol 1984;15: induced

8 Rostom AY, El-Sayed ME. Fat necrosis of the breast. An unusual complication of lumpectomy and radiotherapy in breast cancer. Clin Radiol 1987;38:31.

9 Staples J. Fat necrosis of the breast following radiotherapy. Clin Radiol 1987;38:455-6.

10 Connolly JL, Schnitt SJ. Evaluation of breast biopsy specimens in patients considered for treatment by conservative surgery and radiation therapy for early breast cancer. In: Rosen PP, Fechner RE, eds. Pathology Annual. San Mateo: Appleton and Lange, 1988:1-23.

11 Chaudary MA, Girling A, Girling S, Habib F, Millis RR. New lumps in the breast following conservation treatment for early breast cancer. Br Cancer Treat Res 1988;11:51-8.

12 Rosen PP. Multinucleated mammary stromal giant cells. A benign lesion that simulates invasive carcinoma. Cancer 1979;44:1304-8.

13 Berean K, Tron VA, Churg A, Clement PG. Mammary fibroadenoma with multinucleated stromal giant cells. Am J Surg Pathol 1986;10:823-7.

14 Smith CJ, Echevarra R, McLelland LA. Pseudosarcomatous changes in antrochoanal polyps. Arch Otolaryngol
1974;99:228-30.

15 Bondeson L. Aspiration cytology of radiation-induced changes of normal breast epithelium. Acta Cytol 1987;31 309-10.

16 Peterse JL, Van Heerde P. Radiation induced changes in non-neoplastic breast epithelium. A possible pitfall in cytodiagnosis. In: Hollman KH, Verley JM, eds. New frontiers in mammary pathology. New York: Martinus frontiers in mammary

17 Stomper PC, Recht A Berenberg AL, Jochelson MS, Harris JR. Mammographic detection of recurrent cancer in the irradiated breast. Am J Radiol 1987;148:39-43. 